\title{
GRADED MORITA EQUIVALENCES FOR GEOMETRIC AS-REGULAR ALGEBRAS
}

\author{
IZURU MORI \\ Department of Mathematics, Faculty of Science, Shizuoka University, Shizuoka 422-8529, Japan \\ e-mail:simouri@ipc.shizuoka.ac.jp \\ and KENTA UEYAMA \\ Department of Information Science and Technology, Graduate School of Science and Technology, \\ Shizuoka University, Shizuoka 422-8529, Japan \\ e-mail:f5144004@ipc.shizuoka.ac.jp
}

(Received 12 December 2011; revised 4 April 2012; accepted 2 May 2012;

first published online 2 August 2012)

\begin{abstract}
Classification of AS-regular algebras is one of the major projects in non-commutative algebraic geometry. In this paper, we will study when given ASregular algebras are graded Morita equivalent. In particular, for every geometric AS-regular algebra $A$, we define another graded algebra $\bar{A}$, and show that if two geometric AS-regular algebras $A$ and $A^{\prime}$ are graded Morita equivalent, then $\bar{A}$ and $\overline{A^{\prime}}$ are isomorphic as graded algebras. We also show that the converse holds in many three-dimensional cases. As applications, we apply our results to Frobenius Koszul algebras and Beilinson algebras.
\end{abstract}

2010 Mathematics Subject Classification. 16W50, 16D90, 16S38, 16S37, 16E65.

1. Introduction. Classification of AS-regular algebras is one of the major projects in non-commutative algebraic geometry. In fact, the geometric classification of threedimensional AS-regular algebras due to Artin, Tate and Van den Bergh [2] was the starting point of this field. This paper tries to answer the question when given ASregular algebras are graded Morita equivalent, that is, they have equivalent graded module categories. There are two motivations to answer this question.

The first motivation comes from non-commutative algebraic geometry itself. It is a common understanding that the classification of quantum projective planes was regarded as settled since their homogeneous coordinate rings, namely, threedimensional quadratic AS-regular algebras, were classified [2]. However, it is still interesting to know when two three-dimensional quadratic AS-regular algebras determine isomorphic quantum projective planes in order to say that the classification of quantum projective planes is complete. It was shown in [8, Theorem 5.2] that in many cases, this question is equivalent to the question when two three-dimensional quadratic AS-regular algebras are graded Morita equivalent (see [8] and [19] for details).

The second motivation comes from representation theory of finite-dimensional algebras. It is known that every finite-dimensional algebra of global dimension 1 is a path algebra of a finite acyclic quiver up to Morita equivalence, so such algebras can be classified in terms of quivers. As an obvious next step, it is interesting to classify finite-dimensional algebras of global dimension 2 or higher. Recently, Minamoto 
introduced a nice class of finite-dimensional algebras of finite global dimension, called (quasi-)Fano algebras [6], which are very interesting class of algebras to study and classify. It was shown that for every AS-regular algebra $A$, we can define another algebra $\nabla A$, called the Beilinson algebra associated to $A$, which turns out to be a quasi-Fano algebra [7, Theorem 4.12], and that two AS-regular algebras $A$ and $A^{\prime}$ are graded Morita equivalent if and only if $\nabla A$ and $\nabla A^{\prime}$ are isomorphic as algebras [7, Theorem 4.17]. Moreover, by extending the notion of AS-regularity, every quasiFano algebra can be obtained as a Beilinson algebra of some AS-regular algebra, so the classification of AS-regular algebras up to graded Morita equivalence is strongly related to the classification of quasi-Fano algebras up to isomorphism (see [7] and [11] for details). By this fact, we hope that this paper also appeals to researchers in representation theory of finite-dimensional algebras.

Our main result is as follows. For every geometric AS-regular algebra $A$, we define another graded algebra $\bar{A}$, and show that if two geometric AS-regular algebras $A$ and $A^{\prime}$ are graded Morita equivalent, then $\bar{A}$ and $\overline{A^{\prime}}$ are isomorphic as graded algebras. We also show that the converse holds in many three-dimensional cases. We point out that it is in general much easier to determine if two graded algebras are isomorphic as graded algebras than to determine if they are graded Morita equivalent. We also point out that every three-dimensional quadratic AS-regular algebra is geometric, and most of known examples of four-dimensional quadratic AS-regular algebras are geometric, so there are plenty of examples we can apply our results to. In the last section, we apply our results to Frobenius Koszul algebras and Beilinson algebras.

2. AS-regular algebras. Since this paper is expected to be read by non-experts as well as experts, we will first recall basic definitions and facts in non-commutative algebraic geometry, which are needed in this paper.

Throughout this paper, we fix an algebraically closed field $k$ of characteristic 0 , and we assume that all vector spaces and algebras are over $k$ unless otherwise stated. In this paper, a graded algebra means an $\mathbb{N}$-graded algebra $A=\bigoplus_{i \in \mathbb{N}} A_{i}$. We denote by GrMod $A$ the category of graded right $A$-modules, and by grmod $A$ the full subcategory consisting of finitely generated modules. Morphisms in $\operatorname{GrMod} A$ are right $A$-module homomorphisms preserving degrees. Note that if $A$ is noetherian, then $\operatorname{grmod} A$ is an abelian category. We say that two graded algebras $A$ and $A^{\prime}$ are graded Morita equivalent if $\operatorname{GrMod} A \cong \operatorname{GrMod} A^{\prime}$.

For a graded module $M \in \operatorname{GrMod} A$ and an integer $n \in \mathbb{Z}$, we define the truncation $M_{\geq n}:=\bigoplus_{i \geq n} M_{i} \in \operatorname{GrMod} A$ and the shift $M(n) \in \operatorname{GrMod} A$ by $M(n)_{i}:=M_{n+i}$ for $i \in \mathbb{Z}$. Note that the rule $M \mapsto M(n)$ is a $k$-linear autoequivalence for $\operatorname{GrMod} A$ and $\operatorname{grmod} A$, called the shift functor. For $M, N \in \operatorname{GrMod} A$, we write $\operatorname{Ext}_{A}^{i}(M, N)=$ $\operatorname{Ext}_{\mathrm{GrMod} A}^{i}(M, N)$ and

$$
\underline{\operatorname{Ext}}_{A}^{i}(M, N):=\bigoplus_{n \in \mathbb{Z}} \operatorname{Ext}_{A}^{i}(M, N(n))
$$

We denote by $V^{*}$ the dual vector space of a vector space $V$. If $M$ is a graded right (respectively, left) module over a graded algebra $A$, then we denote by $M^{*}:=$ $\underline{\operatorname{Hom}}_{k}(M, k)$ the dual graded vector space of $M$ by abuse of notation, i.e. $\left(M^{*}\right)_{i}:=$ $\left(M_{-i}\right)^{*}$. Note that $M^{*}$ has a graded left (resp. right) $A$-module structure.

Let $A$ be a noetherian-graded algebra. We denote by tors $A$ the full subcategory of $\operatorname{grmod} A$ consisting of finite-dimensional modules over $k$, and tails $A:=$ 
$\operatorname{grmod} A$ / tors $A$ the quotient category, which is called the non-commutative projective scheme associated to $A$ in [4]. If $A$ is a commutative graded algebra finitely generated in degree 1 over $k$, then tails $A$ is equivalent to the category of coherent sheaves on $\operatorname{Proj} A$ by Serre, justifying the terminology.

We often denote by $\mathcal{M} \in$ tails $A$ the image of $M \in \operatorname{grmod} A$. For $M, N \in \operatorname{grmod} A$, $\mathcal{M} \cong \mathcal{N}$ in tails $A$ if and only if $M_{\geq n} \cong N_{\geq n}$ in $\operatorname{GrMod} A$ for some $n \in \mathbb{Z}$. Note that the $k$-linear autoequivalence $M \mapsto M(n)$ preserves finite-dimensional modules over $k$, so it induces a $k$-linear autoequivalence $\mathcal{M} \mapsto \mathcal{M}(n)$ for tails $A$, again called the shift functor.

A connected graded algebra is a graded algebra $A=\bigoplus_{i \in \mathbb{N}} A_{i}$ such that $A_{0}=k$. For a connected graded algebra $A$, we view $k=A / A_{\geq 1} \in \operatorname{GrMod} A$ as a graded $A$-module. Recall that the Gelfand-Kirillov dimension of $A$ is defined by

$$
\operatorname{GKdim} A:=\inf \left\{d \in \mathbb{R}^{+} \mid \operatorname{dim}_{k} A_{i} \leq c i^{d-1} \text { for some constant } c>0, i \gg 0\right\} .
$$

An AS-regular algebra defined below is one of the main objects of study in noncommutative algebraic geometry.

Definition 2.1. [1]. A connected graded algebra $A$ is called a $d$-dimensional ASregular algebra of Gorenstein parameter $\ell$ if

(1) $\operatorname{gldim} A=d<\infty$,

(2) $\mathrm{GKdim} A<\infty$, and

(3) $\underline{\operatorname{Ext}}_{A}^{i}(k, A) \cong \begin{cases}k(\ell), & \text { if } i=d, \\ 0, & \text { if } i \neq d .\end{cases}$

We now define the generalised Nakayama automorphism for an AS-regular algebra, which plays an essential role in this paper. Let $A$ be a graded algebra, and $\tau \in{\underline{\mathrm{Aut}_{k}}}_{k} A$ a graded algebra automorphism. For a graded right $A$-module $M \in \operatorname{GrMod} A$, we define a new graded right $A$-module $M_{\tau} \in \operatorname{GrMod} A$ by $M_{\tau}=M$ as a graded vector space with the new right action $m * a:=m \tau(a)$ for $m \in M$ and $a \in A$. If $M$ is a graded $A-A$ bimodule, then $M_{\tau}$ is also a graded $A-A$ bimodule by this new right action. The rule $M \mapsto M_{\tau}$ is a $k$-linear autoequivalence for $\operatorname{GrMod} A$ and $\operatorname{grmod} A$. If $A$ is noetherian, then $(-)_{\tau} \cong-\otimes_{A} A_{\tau} \in$ Aut $_{k}(\operatorname{grmod} A)$ commutes with the shift functor $(n) \in \operatorname{Aut}_{k}(\operatorname{grmod} A)$ and preserves tors $A$, so it induces a $k$-linear autoequivalence $(-)_{\tau} \cong-\otimes_{\mathcal{A}} \mathcal{A}_{\tau} \in \operatorname{Aut}_{k}($ tails $A$ ) commuting with the shift functor (n) $\in$ Aut $_{k}(\operatorname{tails} A)$.

Let $A$ be a connected graded algebra and $\mathfrak{m}=A_{\geq 1}$ the maximal homogeneous two-sided ideal of $A$. For $M \in \operatorname{GrMod} A$, we define the $i$-th local cohomology of $M$ by

$$
\underline{\mathrm{H}}_{\mathfrak{m}}^{i}(M):=\lim _{n \rightarrow \infty} \underline{\operatorname{Ext}}_{A}^{i}\left(A / A_{\geq n}, M\right) \in \operatorname{GrMod} A .
$$

If $A$ is a noetherian $d$-dimensional AS-regular algebra of Gorenstein parameter $\ell$, then $\underline{\mathrm{H}}_{\mathfrak{m}}^{i}(A)=0$ for all $i \neq d$. The graded $A$-A bimodule $\omega_{A}:=\underline{\mathrm{H}}_{\mathfrak{m}}^{d}(A)^{*}$ is called the canonical module of $A$. It is known that there exists a graded algebra automorphism $v \in \underline{\text { Aut }}_{k} A$ such that $\omega_{A} \cong A_{v^{-1}}(-\ell)$ as graded $A$ - $A$ bimodules (c.f. [5, Theorem 1.2]). We call this graded algebra automorphism $v \in \mathrm{Aut}_{k} A$ the generalised Nakayama automorphism of $A$. The canonical module induces the Serre functor defined below.

Definition 2.2. Let $\mathcal{C}$ be a $k$-linear category such that $\operatorname{dim}_{k} \operatorname{Hom}_{\mathcal{C}}(\mathcal{M}, \mathcal{N})<\infty$ for all $\mathcal{M}, \mathcal{N} \in \mathcal{C}$. An autoequivalence $\mathcal{S} \in \mathrm{Aut}_{k} \mathcal{C}$ is called the Serre functor for $\mathcal{C}$ if 
we have a functorial isomorphism

$$
\operatorname{Hom}_{\mathcal{C}}(\mathcal{M}, \mathcal{N}) \cong \operatorname{Hom}_{\mathcal{C}}(\mathcal{N}, \mathcal{S}(\mathcal{M}))^{*}
$$

for all $\mathcal{M}, \mathcal{N} \in \mathcal{C}$.

Note that the Serre functor is unique if it exists. Let $A$ be a noetherian $d$-dimensional AS-regular algebra with the canonical module $\omega_{A}$. Since $\omega_{A} \cong$ $A_{v^{-1}}(-\ell)$, where $v \in$ Aut $_{k} A$ is the generalised Nakayama automorphism, $-\otimes_{A} \omega_{A} \cong$ $(-)_{v^{-1}}(-\ell) \in$ Aut $_{k}(\operatorname{grmod} A)$ induces an autoequivalence $-\otimes_{\mathcal{A}}^{\mathbf{L}} \omega_{\mathcal{A}} \in$ Aut $_{k} \mathcal{D}^{b}($ tails $A)$, where $\mathcal{D}^{b}$ (tails $A$ ) denotes the bounded derived category of tails $A$. It was shown in [13] that $-\otimes_{\mathcal{A}}^{\mathbf{L}} \omega_{\mathcal{A}}[d-1]$ is the Serre functor for $\mathcal{D}^{b}($ tails $A)$.

We will recall below geometric techniques to classify three-dimensional quadratic AS-regular algebras due to Artin, Tate and Van den Bergh [2]. Note that every graded algebra finitely generated in degree 1 over $k$ can be presented as $A=T(V) / I$, where $V$ is a finite-dimensional vector space, $T(V)$ is the tensor algebra on $V$ over $k$ and $I$ is a homogeneous two-sided ideal of $T(V)$. Let $A=T(V) /(R)$ be a quadratic algebra, that is, $R \subset V \otimes_{k} V$ is a subspace and $(R)$ is the two-sided ideal of $T(V)$ generated by $R$. Since every element $f \in R \subset V \otimes_{k} V$ defines a bilinear form $f: V^{*} \otimes_{k} V^{*} \cong$ $\left(V \otimes_{k} V\right)^{*} \rightarrow k$, we can define

$$
\mathcal{V}(R):=\left\{(p, q) \in \mathbb{P}\left(V^{*}\right) \times \mathbb{P}\left(V^{*}\right) \mid f(p, q)=0 \text { for all } f \in R\right\} .
$$

Definition 2.3. Let $A=T(V) /(R)$ be a quadratic algebra. A geometric pair $(E, \sigma)$ consists of a subscheme $E \subset \mathbb{P}\left(V^{*}\right)$ and an automorphism $\sigma \in$ Aut $_{k} E$.

(1) We say that $A$ satisfies (G1) if there exists a geometric pair $(E, \sigma)$ such that

$$
\mathcal{V}(R)=\left\{(p, \sigma(p)) \in \mathbb{P}\left(V^{*}\right) \times \mathbb{P}\left(V^{*}\right) \mid p \in E\right\} .
$$

In this case, we write $P(A)=(E, \sigma)$.

(2) We say that $A$ satisfies (G2) if there exists a geometric pair $(E, \sigma)$ such that

$$
R=\left\{f \in V \otimes_{k} V \mid f(p, \sigma(p))=0 \text { for all } p \in E\right\} .
$$

In this case, we write $A=A(E, \sigma)$.

(3) A noetherian quadratic algebra $A$ is called geometric if $A$ satisfies both (G1) and (G2) with $A \cong A(P(A))$.

Note that every three-dimensional quadratic AS-regular algebra is geometric, and most of known examples of four-dimensional quadratic AS-regular algebras are geometric. As a typical example, a four-dimensional Sklyanin algebra is a geometric AS-regular algebra. However, it was shown in [18] that there exists a family of fourdimensional AS-regular algebras that do not satisfy (G2). As far as we know, there exists no example of an AS-regular algebra that does not satisfy (G1).

The purpose of this paper is to provide a way to determine when geometric ASregular algebras are graded Morita equivalent. The notion of twisting system defined below plays an essential role for graded Morita equivalence.

Definition 2.4. [20]. Let $A$ be a graded algebra. A twisting system $\theta$ of $A$ is a sequence $\left\{\theta_{i}\right\}$ of graded vector space automorphisms of $A$ such that $\theta_{l}\left(a \theta_{m}(b)\right)=$ $\theta_{l}(a) \theta_{l+m}(b)$ for all $l, m, n \in \mathbb{Z}$ and all $a \in A_{m}, b \in A_{n}$. 
Let $\theta$ be a twisting system of a graded algebra $A$. We define a new graded algebra $A^{\theta}$, called a twist of $A$, by $A^{\theta}=A$ as a graded vector space with the new multiplication $a *$ $b=a \theta_{m}(b)$ for all $a \in A_{m}, b \in A_{n}$. Similarly, for $M \in \operatorname{GrMod} A$, we define a new graded right $A^{\theta}$-module $M^{\theta}$ by $M^{\theta}=M$ as a graded vector space with the new right action $x *$ $a=x \theta_{m}(a)$ for all $x \in M_{m}, a \in A_{n}^{\theta}$. The rule $M \mapsto M^{\theta}$ defines an equivalence functor $\operatorname{GrMod} A \rightarrow \operatorname{GrMod} A^{\theta}$. Conversely, every graded Morita equivalence is obtained in this way (see [20] for details). Note that every graded algebra automorphism $\tau \in \underline{\mathrm{Aut}}_{k} A$ defines a twisting system $\left\{\tau^{i}\right\}$ of $A$. In this case, we write $A^{\tau}:=A^{\left\{\tau^{i}\right\}}$.

Geometric pairs can be used to classify geometric algebras by the following lemmas. Since these lemmas are refinements of [8, Theorem 4.7], we will leave the proofs to the reader. If quadratic algebras $A=T(V) /(R), A^{\prime}=T\left(V^{\prime}\right) /\left(R^{\prime}\right)$ are graded Morita equivalent, then $V \cong V^{\prime}$ by [20], so we may assume that $V=V^{\prime}$ in the lemma below.

LEMMA 2.5. Let $A=T(V) /(R)$ and $A^{\prime}=T(V) /\left(R^{\prime}\right)$ be quadratic algebras satisfying (G1) with $P(A)=(E, \sigma), P\left(A^{\prime}\right)=\left(E^{\prime}, \sigma^{\prime}\right)$.

(1) If $A \cong A^{\prime}$ as graded algebras, then there exists an automorphism $\tau$ of $\mathbb{P}\left(V^{*}\right)$ which restricts to an isomorphism $\tau: E \rightarrow E^{\prime}$ such that

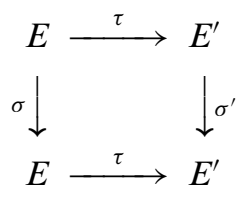

commutes.

(2) If $\operatorname{GrMod} A \cong \operatorname{GrMod} A^{\prime}$, then there exists a sequence of automorphisms $\tau_{n}$ of $\mathbb{P}\left(V^{*}\right)$ which restrict to isomorphisms $\tau_{n}: E \rightarrow E^{\prime}$ such that

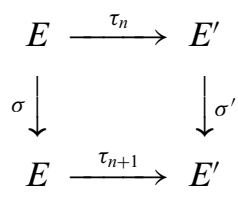

commute for all $n \in \mathbb{Z}$.

LEMMA 2.6. Let $A=T(V) /(R)$ and $A^{\prime}=T(V) /\left(R^{\prime}\right)$ be quadratic algebras satisfying (G2) with $A=A(E, \sigma), A^{\prime}=A\left(E^{\prime}, \sigma^{\prime}\right)$.

(1) If there exists an automorphism $\tau$ of $\mathbb{P}\left(V^{*}\right)$ which restricts to an isomorphism $\tau: E \rightarrow E^{\prime}$ such that

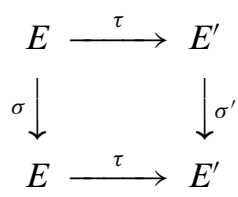

commutes, then $A \cong A^{\prime}$ as graded algebras. 
(2) If there exists a sequence of automorphisms $\tau_{n}$ of $\mathbb{P}\left(V^{*}\right)$ which restrict to isomorphisms $\tau_{n}: E \rightarrow E^{\prime}$ such that

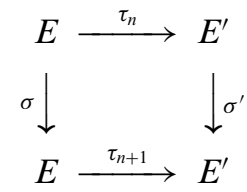

commute for all $n \in \mathbb{Z}$, then $\operatorname{GrMod} A \cong \operatorname{GrMod} A^{\prime}$.

In [2], three-dimensional quadratic AS-regular algebras were classified in terms of geometric pairs. (In the classification, $E \subset \mathbb{P}\left(V^{*}\right)$ could be a non-reduced subscheme and in this case, we need to modify the definition of a geometric algebra, for which we refer to [8, Definition 4.3].) For the purpose of this paper, we define the types of (reduced and reducible) geometric pairs $(E, \sigma)$ of three-dimensional quadratic ASregular algebras $A=A(E, \sigma)$, slightly modifying and extending the types defined in [1] and [2].

- Type $\mathbb{P}^{2}: E$ is $\mathbb{P}^{2}$, and $\sigma \in \operatorname{Aut}_{k} \mathbb{P}^{2}=\operatorname{PGL}_{3}(k)$.

- Type $S_{1}: E$ is a triangle, and $\sigma$ stabilises each component.

- Type $S_{2}: E$ is a triangle, and $\sigma$ interchanges two of its components.

- Type $S_{3}: E$ is a triangle, and $\sigma$ circulates three components.

- Type $S_{1}^{\prime}: E$ is a union of a line and a conic meeting at two points, and $\sigma$ stabilises each component and two intersection points.

- Type $S_{2}^{\prime}: E$ is a union of a line and a conic meeting at two points, and $\sigma$ stabilises each component and interchanges two intersection points.

- Type $T_{1}: E$ is a union of three lines meeting at one point, and $\sigma$ stabilises each component.

- Type $T_{2}: E$ is a union of three lines meeting at one point, and $\sigma$ interchanges two of its components.

- Type $T_{3}: E$ is a union of three lines meeting at one point, and $\sigma$ circulates three components.

- Type $T_{1}^{\prime}: E$ is a union of a line and a conic meeting at one point, and $\sigma$ stabilises each component.

Let $A=A(E, \sigma)$ be a three-dimensional quadratic AS-regular algebra. If $E$ is a union of a line and a conic and $\sigma$ interchanges these two components, then $A(E, \sigma)$ is not AS-regular by [2, Proposition 4.11]. It follows that if $E$ is a reduced and reducible cubic in $\mathbb{P}^{2}$, then $(E, \sigma)$ is one of the above types.

3. Main results. In general, it is easier to determine if two graded algebras are isomorphic as graded algebras than to determine if they are graded Morita equivalent. In this section, for a geometric AS-regular algebra $A$, we will define a new graded algebra $\bar{A}$ and compare the condition (A) GrMod $A \cong$ GrMod $A^{\prime}$ and the condition (B) $\bar{A} \cong \overline{A^{\prime}}$ as graded algebras.

3.1. The general case. A point module defined below plays an important role to study a graded algebra in non-commutative algebraic geometry.

Definition 3.1. [2]. Let $A$ be a graded algebra finitely generated in degree 1 over $k$. A graded module $M \in \operatorname{GrMod} A$ is called a point module if 
(1) $M$ is cyclic, and

(2) $\operatorname{dim}_{k} M_{i}= \begin{cases}1, & \text { if } i \geq 0 \\ 0, & \text { if } i<0\end{cases}$

Note that if $M$ is a point module, then $M(i)_{\geq 0}$ are also point modules for all $i \in \mathbb{N}$. Let $A=T(V) / I$ be a graded algebra finitely generated in degree 1 . For a point $p \in \mathbb{P}\left(V^{*}\right)$, define a graded module $M_{p}:=A / p^{\perp} A \in \operatorname{GrMod} A$ where $p^{\perp}:=\left\{f \in A_{1}=\right.$ $V \mid f(p)=0\}$. It is easy to see that for points $p, q \in \mathbb{P}\left(V^{*}\right), M_{p} \cong M_{q}$ if and only if $p=q$.

Every point module is isomorphic to $M_{p}$ for some $p \in \mathbb{P}\left(V^{*}\right)$, but the module of the form $M_{p}$ is not always a point module. Suppose that $A$ satisfies $(\mathbf{G 1})$ with $P(A)=(E, \sigma)$. Then $M_{p}$ is a point module for any point $p \in E$. Moreover, by [9, Lemma 2.5], there is a bijection between the set of (closed) points of $E$ and isomorphism classes of point modules via $p \mapsto M_{p}$, and $\sigma \in$ Aut $_{k} E$ is given by $M_{\sigma(p)} \cong M_{p}(1)_{\geq 0}$.

Note that if $A=T(V) / I$ is a graded algebra finitely generated in degree 1 , then every graded algebra automorphism $\tau \in \underline{\text { Aut }}_{k} A$ is uniquely determined by $\left.\tau\right|_{A_{1}}=\left.\tau\right|_{V} \in$ $\mathrm{GL}(V)$. If $\tau \in \mathrm{Aut}_{k} A$ is a graded algebra automorphism, then the dual of the restriction $\left.\tau\right|_{V}=\left.\tau\right|_{A_{1}}$ induces an automorphism $\tau^{*} \in \operatorname{Aut}_{k} \mathbb{P}\left(V^{*}\right)$. It is easy to see that $M \in$ $\operatorname{GrMod} A$ is a point module if and only if $M_{\tau} \in \operatorname{GrMod} A$ is a point module. By [10, Lemma 3.2], $\left(M_{p}\right)_{\tau} \cong M_{\tau^{*}(p)}$ for $p \in \mathbb{P}\left(V^{*}\right)$, so $\tau^{*}$ restricts to an automorphism $\tau^{*} \in \mathrm{Aut}_{k} E$ by abuse of notation. Moreover,

$$
\begin{aligned}
M_{\sigma \tau^{*}(p)} \cong M_{\tau^{*}(p)}(1)_{\geq 0} \cong\left(M_{p}\right)_{\tau}(1)_{\geq 0} \\
\cong\left(M_{p}(1)_{\tau}\right)_{\geq 0} \cong\left(M_{p}(1)_{\geq 0}\right)_{\tau} \\
\cong\left(M_{\sigma(p)}\right)_{\tau} \cong M_{\tau^{*} \sigma(p)},
\end{aligned}
$$

for $p \in E$, so $\tau^{*}$ commutes with $\sigma$ on $E$. We can now define a new graded algebra $\bar{A}$ as below.

DeFINITION 3.2. Let $A$ be an AS-regular algebra of Gorenstein parameter $\ell$ with the generalised Nakayama automorphism $v \in{\underline{\mathrm{Aut}_{k}}}_{k} A$. If $A$ satisfies $(\mathbf{G 1})$ with $P(A)=$ $(E, \sigma)$, then we define a new graded algebra satisfying $(\mathbf{G 2})$ by $\bar{A}:=A\left(E, v^{*} \sigma^{\ell}\right)$.

We now prepare two easy lemmas.

LEMMA 3.3. Let $A=T(V) /(R)$ and $A^{\prime}=T(V) /\left(R^{\prime}\right)$ be quadratic algebras satisfying (G1) with $P(A)=(E, \sigma), P\left(A^{\prime}\right)=\left(E^{\prime}, \sigma^{\prime}\right)$. If $\operatorname{GrMod} A \cong \operatorname{GrMod} A^{\prime}$, then there exists an equivalence functor $F: \operatorname{GrMod} A \rightarrow \operatorname{GrMod} A^{\prime}$ which induces an isomorphism $\tau$ : $E \rightarrow E^{\prime}$ by $F\left(M_{p}\right) \cong M_{\tau(p)}$ for $p \in E$. Moreover, $\tau$ extends to an automorphism $\bar{\tau} \in$ $\operatorname{Aut}_{k} \mathbb{P}\left(V^{*}\right)$.

Proof. If $A$ and $A^{\prime}$ are quadratic algebras such that $\operatorname{GrMod} A \cong \operatorname{GrMod} A^{\prime}$, then there exists a twisting system $\theta$ of $A$ such that $A^{\prime} \cong A^{\theta}$ as graded algebras by [20, Theorem 1.2], so we may assume that $A^{\prime}=A^{\theta}$. We leave the reader to check that the equivalence functor $F: \operatorname{GrMod} A \rightarrow \operatorname{GrMod} A^{\theta}$ defined by $F(M)=M^{\theta}$ satisfies the desired properties.

Lemma 3.4. Let $A$ be a noetherian quadratic algebra satisfying (G1) with $P(A)=$ $(E, \sigma)$. For points $p, q \in E, \mathcal{M}_{p} \cong \mathcal{M}_{q}$ in tails $A$ if and only if $p=q$. 
Proof. If $p=q$, then clearly $\mathcal{M}_{p} \cong \mathcal{M}_{q}$. Conversely, if $\mathcal{M}_{p} \cong \mathcal{M}_{q}$, then

$$
M_{\sigma^{i}(p)} \cong M_{p}(i)_{\geq 0} \cong\left(M_{p}\right)_{\geq i}(i) \cong\left(M_{q}\right)_{\geq i}(i) \cong M_{q}(i)_{\geq 0} \cong M_{\sigma^{i}(q)},
$$

for some $i \in \mathbb{N}$, so $\sigma^{i}(p)=\sigma^{i}(q)$. Since $\sigma \in \mathrm{Aut}_{k} E$ is an automorphism, $p=q$.

The theorem below shows that $(\mathbf{A}) \Rightarrow$ (B) holds for general geometric AS-regular algebras.

THEOREM 3.5. Let $A$ and $A^{\prime}$ be noetherian quadratic AS-regular algebras satisfying (G1). If (A) GrMod $A \cong \operatorname{GrMod} A^{\prime}$, then (B) $\bar{A} \cong \overline{A^{\prime}}$ as graded algebras.

Proof. Let $A=T(V) /(R), A^{\prime}=T(V) /\left(R^{\prime}\right), P(A)=(E, \sigma), P\left(A^{\prime}\right)=\left(E^{\prime}, \sigma^{\prime}\right)$ with Gorenstein parameters $\ell$ and $\ell^{\prime}$ and the generalised Nakayama automorphisms $v \in \operatorname{Aut}_{k} A, v^{\prime} \in \operatorname{Aut}_{k} A^{\prime}$. If $\operatorname{GrMod} A \cong \operatorname{GrMod} A^{\prime}$, then $\operatorname{gldim} A=\operatorname{gldim} A^{\prime}=: d$ by [20, Theorem 1.3]. By Lemma 3.3, there exists an equivalence functor $F: \operatorname{GrMod} A \rightarrow$ GrMod $A^{\prime}$ which induces an isomorphism $\tau: E \rightarrow E^{\prime}$ by $F\left(M_{p}\right) \cong M_{\tau(p)}$ for $p \in E$. Moreover, the equivalence functor $F: \operatorname{GrMod} A \rightarrow \operatorname{GrMod} A^{\prime}$ induces a triangle equivalence functor $\bar{F}: \mathcal{D}^{b}($ tails $A) \rightarrow \mathcal{D}^{b}$ (tails $\left.A^{\prime}\right)$, so we have a commutative diagram

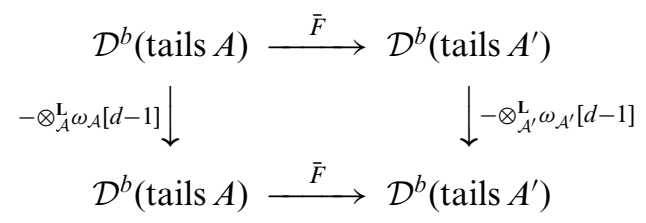

by uniqueness of the Serre functor. Since $\bar{F}$ commutes with the suspension functor $[d-1]$, we have a commutative diagram

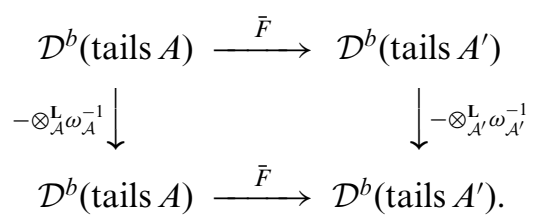

Since

$$
\left(M_{p} \otimes_{A} \omega_{A}^{-1}\right)_{\geq 0} \cong\left(M_{p}\right)_{\nu}(\ell)_{\geq 0} \cong M_{\nu^{*}(p)}(\ell)_{\geq 0} \cong M_{\sigma^{\ell} \nu^{*}(p)} \cong M_{\nu^{*} \sigma^{\ell}(p)}
$$

for $p \in E$,

$$
\mathcal{M}_{p} \otimes_{\mathcal{A}}^{\mathbf{L}} \omega_{\mathcal{A}}^{-1} \cong \mathcal{M}_{p} \otimes_{\mathcal{A}} \omega_{\mathcal{A}}^{-1} \cong \mathcal{M}_{\nu^{*} \sigma^{\ell}(p)} .
$$

By Lemma 3.4, the above commutative diagram induces a commutative diagram

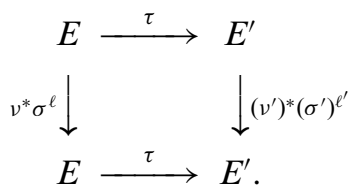

Since $\tau$ extends to an automorphism $\bar{\tau} \in \operatorname{Aut}_{k} \mathbb{P}\left(V^{*}\right)$ by Lemma $3.3, \bar{A} \cong \overline{A^{\prime}}$ as graded algebras by Lemma 2.6 . 
3.2. The three-dimensional case. Unfortunately, there are examples of fourdimensional geometric AS-regular algebras such that $(\mathbf{B}) \Rightarrow(\mathbf{A})$ does not hold [16, Example 4.2]. On the other hand, $(\mathbf{B}) \Rightarrow(\mathbf{A})$ has been shown to hold for many threedimensional geometric AS-regular algebras $[\mathbf{8}, \mathbf{1 6}]$. In this subsection, we will prove that (B) $\Rightarrow$ (A) holds for several more cases. In the proof of the theorem below, we will see that the geometric technique is useful to determine graded Morita equivalence.

TheOREM 3.6. Let $A=A(E, \sigma)$ and $A^{\prime}=A\left(E^{\prime}, \sigma^{\prime}\right)$ be three-dimensional quadratic $A S$-regular algebras with the generalised Nakayama automorphisms $v \in \underline{\operatorname{Aut}}_{k} A, v^{\prime} \in$ Aut $_{k} A^{\prime}$. Suppose that $(E, \sigma)$ and $\left(E^{\prime}, \sigma^{\prime}\right)$ are of the same type $\mathbb{P}^{2}, S_{1}, S_{1}^{\prime}, T_{1}$ or $T_{1}^{\prime}$. Then (A) $\operatorname{GrMod} A \cong \operatorname{GrMod} A^{\prime}$ if and only if (B) $\bar{A} \cong \overline{A^{\prime}}$ as graded algebras.

Proof. For the types $\mathbb{P}^{2}, S_{1}, S_{1}^{\prime}$, the result follows from [16, Theorem 3.1], so it remains to show it for the types $T_{1}$ and $T_{1}^{\prime}$. By Theorem 3.5, it is enough to show (B) $\Rightarrow$ (A). In fact, we can show that (A) always holds for these two types. We will show this fact for the type $T_{1}$, and leave a proof for the type $T_{1}^{\prime}$ to the reader.

If $(E, \sigma)$ and $\left(E^{\prime}, \sigma^{\prime}\right)$ are of type $T_{1}$, then we may assume that $E=l_{1} \cup l_{2} \cup l_{3}$ where $l_{1}=\mathcal{V}(x), l_{2}=\mathcal{V}(y), l_{3}=\mathcal{V}(x-y)$ and $\sigma \in \mathrm{Aut}_{k} E$ is given by

$$
\begin{aligned}
& \left.\sigma\right|_{l_{1}}(0, b, c)=(0, b, \alpha b+\delta c), \\
& \left.\sigma\right|_{l_{2}}(a, 0, c)=(a, 0, \beta a+\delta c), \\
& \left.\sigma\right|_{l_{3}}(a, a, c)=(a, a,-\gamma a+\delta c),
\end{aligned}
$$

where $\alpha+\beta+\gamma \neq 0, \delta \neq 0$. In this case, $A=A(E, \sigma)$ is given by

$$
A=k\langle x, y, z\rangle /\left(y z-\delta z y-\alpha y^{2}+(\alpha+\gamma) y x, x z-\delta z x-\beta x^{2}+(\beta+\gamma) x y, x y-y x\right) .
$$

By a Hilbert series calculation, we can show that $\delta=1$ is necessary for $A$ to be a three-dimensional quadratic AS-regular algebra. Similarly, we may assume that $E^{\prime}=l_{1} \cup l_{2} \cup l_{3}$ and $\sigma^{\prime} \in \mathrm{Aut}_{k} E^{\prime}$ is given by

$$
\begin{aligned}
& \left.\sigma\right|_{l_{1}}(0, b, c)=\left(0, b, \alpha^{\prime} b+c\right), \\
& \left.\sigma\right|_{l_{2}}(a, 0, c)=\left(a, 0, \beta^{\prime} a+c\right), \\
& \left.\sigma\right|_{l_{3}}(a, a, c)=\left(a, a,-\gamma^{\prime} a+c\right),
\end{aligned}
$$

where $\alpha+\beta+\gamma \neq 0$. For $n \in \mathbb{Z}$, we define $\tau_{n} \in$ Aut $_{k} E$ by

$$
\begin{aligned}
\left.\tau_{n}\right|_{l_{1}}(0, b, c) & =\left(0, b, \frac{n\left(-\lambda^{\prime} \alpha+\lambda \alpha^{\prime}\right)}{\lambda} b+\frac{\lambda^{\prime}}{\lambda} c\right), \\
\left.\tau_{n}\right|_{l_{2}}(a, 0, c) & =\left(a, 0, \frac{n\left(-\lambda^{\prime} \beta+\lambda \beta^{\prime}\right)}{\lambda} a+\frac{\lambda^{\prime}}{\lambda} c\right), \\
\left.\tau_{n}\right|_{l_{3}}(a, a, c) & =\left(a, a, \frac{n\left(-\lambda^{\prime}(\alpha+\beta)+\lambda\left(\alpha^{\prime}+\beta^{\prime}\right)\right)}{\lambda} a+\frac{\lambda^{\prime}}{\lambda} c\right),
\end{aligned}
$$

where $\lambda:=\alpha+\beta+\gamma, \lambda^{\prime}:=\alpha^{\prime}+\beta^{\prime}+\gamma^{\prime}$. It is easy to check that $\tau_{n}$ extend to

$$
\bar{\tau}_{n}=\left(\begin{array}{ccc}
1 & 0 & 0 \\
0 & 1 & 0 \\
\frac{n\left(-\lambda^{\prime} \beta+\lambda \beta^{\prime}\right)}{\lambda} & \frac{n\left(-\lambda^{\prime} \alpha+\lambda \alpha^{\prime}\right)}{\lambda} & \frac{\lambda^{\prime}}{\lambda}
\end{array}\right) \in \mathrm{PGL}_{3}(k)=\operatorname{Aut}_{k} \mathbb{P}^{2},
$$


and that $\tau_{n}$ induce the commutative diagrams

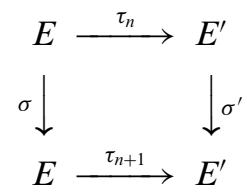

for all $n \in \mathbb{Z}$, so it follows from Lemma 2.6 that (A) GrMod $A \cong \operatorname{GrMod} A^{\prime}$ holds for any $A, A^{\prime}$.

We will now extend the above theorem for any reduced and reducible cubic $E$ in $\mathbb{P}^{2}$. Let $A$ be a graded algebra. Recall that an element $w \in A$ is called regular if $a w=0$ implies $a=0$ and $w a=0$ implies $a=0$. An element $w \in A$ is called normalising if $A w=w A$. If $w \in A_{i}$ is a homogeneous regular normalising element, then the map $\tau_{w}: A \rightarrow A$ defined by $w \tau_{w}(a)=a w$ is a graded algebra automorphism of $A$.

Let $A$ and $A^{\prime}$ be connected graded algebras such that $\operatorname{GrMod} A \cong \operatorname{GrMod} A^{\prime}$. Then $A$ is a three-dimensional quadratic AS-regular algebra if and only if so is $A^{\prime}$ by [20, Theorems 1.2 and 1.3].

LEMMA 3.7. Let $A=A(E, \sigma)$ be a three-dimensional quadratic AS-regular algebra. If $(E, \sigma)$ is of type $X_{2}$, then there exists a homogeneous regular normalising element $w \in A_{1}$ such that $A^{\tau_{w}}$ is a three-dimensional geometric AS-regular algebra of type $X_{1}$ where $X=S, S^{\prime}$ or $T$.

Proof. First note that $A$ is a domain by [3, Theorem 3.9], so every element of $A$ is regular. We will give a proof for the type $T_{2}$, and leave the rest to the reader. If $(E, \sigma)$ is of type $T_{2}$, then we may assume that $E=l_{1} \cup l_{2} \cup l_{3}$ where $l_{1}=\mathcal{V}(x), l_{2}=\mathcal{V}(y), l_{3}=$ $\mathcal{V}(x-y), \sigma \in \mathrm{Aut}_{k} E$ is given by

$$
\begin{aligned}
& \left.\sigma\right|_{l_{1}}(0, b, c)=(b, 0, \alpha b+c), \\
& \left.\sigma\right|_{l_{2}}(a, 0, c)=(0, a, \beta a+c), \\
& \left.\sigma\right|_{l_{3}}(a, a, c)=(a, a,-\gamma a+c),
\end{aligned}
$$

where $\alpha+\beta+\gamma \neq 0$, and so $A=A(E, \sigma)$ is given by $k\langle x, y, z\rangle$ with the defining relations

$$
y z-z x-\alpha y x+(\alpha+\gamma) y^{2}, \quad x z-z y-\beta x y+(\beta+\gamma) x^{2}, \quad x^{2}-y^{2} .
$$

It follows from

$$
\begin{aligned}
& x(x-y)=(x-y)(-y), \\
& y(x-y)=(x-y)(-x), \\
& z(x-y)=(x-y)(\alpha x+\beta y-z)
\end{aligned}
$$

that $x-y$ is normalising, so $\tau:=\tau_{x-y}$ defined by

$$
\tau(x)=-y, \quad \tau(y)=-x, \quad \tau(z)=\alpha x+\beta y-z
$$


is a graded algebra automorphism of $A$. Twisting by $\tau$ yields that $A^{\tau}$ is $k\langle x, y, z\rangle$ with the defining relations

$$
y z-z y+(\alpha+\beta+\gamma) y x, \quad x z-z x+(\alpha+\beta+\gamma) x y, \quad x y-y x,
$$

which is a three-dimensional geometric AS-regular algebra of type $T_{1}$.

LemMA 3.8. Let $A=A(E, \sigma)$ be a three-dimensional quadratic AS-regular algebra. If $(E, \sigma)$ is of type $X_{i}$, then there exists a three-dimensional geometric AS-regular algebra $A^{\prime}=A\left(E^{\prime}, \sigma^{\prime}\right)$ of type $X_{1}$ such that $\operatorname{GrMod} A \cong \operatorname{GrMod} A^{\prime}$, where $X=S, S^{\prime}, T$ and $i=1,2,3$.

Proof. For types $S_{2}, S_{2}^{\prime}$ and $T_{2}$, the result follows from Lemma 3.7, and for the type $S_{3}$, the result follows from [14, Lemma 1.5], so it remains to show it for type $T_{3}$. But the result for this type follows quickly by using Lemma 2.6 (2), so we leave the proof to the reader.

TheOREM 3.9. Let $A=A(E, \sigma)$ and $A^{\prime}=A\left(E^{\prime}, \sigma^{\prime}\right)$ be three-dimensional quadratic $A S$-regular algebras such that $E \cong E^{\prime}$. Suppose that $E=\mathbb{P}^{2}$ or $E$ is a reduced and reducible cubic in $\mathbb{P}^{2}$. Then (A) GrMod $A \cong \operatorname{GrMod} A^{\prime}$ if and only if (B) $\bar{A} \cong \overline{A^{\prime}}$ as graded algebras.

Proof. By Theorem 3.5, it is enough to show (B) $\Rightarrow$ (A). If $E \cong E^{\prime} \cong \mathbb{P}^{2}$, then the result follows from Theorem 3.6. Otherwise, $(E, \sigma)$ and $\left(E^{\prime}, \sigma^{\prime}\right)$ are of types $X_{i}, X_{j}$ for $X=S, S^{\prime}, T, T^{\prime}$ and $i, j=1,2,3$, so there exist three-dimensional geometric ASregular algebras $B$ and $B^{\prime}$ of the same type $X_{1}$ such that $\operatorname{GrMod} A \cong \operatorname{GrMod} B$ and $\operatorname{GrMod} A^{\prime} \cong \operatorname{GrMod} B^{\prime}$ by Lemma 3.8. By Theorem $3.5, \bar{B} \cong \bar{A} \cong \overline{A^{\prime}} \cong \overline{B^{\prime}}$, so $\operatorname{GrMod} A \cong \operatorname{GrMod} B \cong \operatorname{GrMod} B^{\prime} \cong \operatorname{GrMod} A^{\prime}$ by Theorem 3.6.

4. Applications. In this last section, we will apply the results in the previous section to Frobenius Koszul algebras and Beilinson algebras.

4.1. Frobenius Koszul algebras. First, we apply our results to Frobenius Koszul algebras. A graded algebra $A$ is called $h$-homogeneous if $A \cong T(V) /(R)$, where $R \subset$ $V^{\otimes h}$ is a subspace for some $h \geq 2$ and $(R)$ is the two-sided ideal of $T(V)$ generated by $R$. If $A=T(V) /(R)$ is an $h$-homogeneous algebra, then we define the dual graded algebra by $A^{!}:=T\left(V^{*}\right) /\left(R^{\perp}\right)$, where

$$
R^{\perp}:=\left\{\lambda \in\left(V^{*}\right)^{\otimes h} \cong\left(V^{\otimes h}\right)^{*} \mid \lambda(r)=0 \text { for all } r \in R\right\} .
$$

Clearly, $A^{!}$is again an $h$-homogeneous algebra and $\left(A^{!}\right)^{!} \cong A$ as graded algebras. The following result may be known. Due to the lack of a suitable reference, we will include a proof for the second statement.

Lemma 4.1. Let $A=T(V) /(R)$ and $A^{\prime}=T(V) /\left(R^{\prime}\right)$ be h-homogeneous algebras.

(1) $A \cong A^{\prime}$ as graded algebras if and only if $A^{!} \cong\left(A^{\prime}\right)^{!}$as graded algebras.

(2) $\operatorname{GrMod} A \cong \operatorname{GrMod} A^{\prime}$ if and only if $\operatorname{GrMod} A^{!} \cong \operatorname{GrMod}\left(A^{\prime}\right)$.

Proof. We will give a proof for (2). Suppose that $\operatorname{GrMod} A \cong \operatorname{GrMod} A^{\prime}$. Since $A^{\prime}$ is isomorphic to a twist of $A$, there exist graded vector space isomorphisms 
$\phi_{n}: A^{\prime} \rightarrow A$ such that $\phi_{n}(a b)=\phi_{n}(a) \phi_{n+l}(b)$ for all $l, m, n \in \mathbb{Z}$ and $a \in A_{l}^{\prime}, b \in A_{m}^{\prime}$ by [20, Proposition 2.8]. We define graded vector space isomorphisms $\phi_{n}: T(V) \rightarrow T(V)$ by

$$
\left.\phi_{n}\right|_{V \otimes l}\left(v_{1} \otimes v_{2} \otimes \cdots \otimes v_{l}\right)=\left.\left.\left.\phi_{n}\right|_{V}\left(v_{1}\right) \otimes \phi_{n+1}\right|_{V}\left(v_{2}\right) \otimes \cdots \otimes \phi_{n+l-1}\right|_{V}\left(v_{l}\right) .
$$

Let $\left.\psi_{n}\right|_{V^{*}}: V^{*} \rightarrow V^{*}$ be isomorphisms induced by the dual of $\left.\phi_{n}\right|_{V}$. Define graded vector space isomorphisms $\psi_{n}: T\left(V^{*}\right) \rightarrow T\left(V^{*}\right)$ by

$$
\left.\psi_{n}\right|_{\left(V^{*}\right)^{\otimes l}}\left(\xi_{1} \otimes \xi_{2} \otimes \cdots \otimes \xi_{l}\right)=\left.\left.\left.\psi_{n}\right|_{V^{*}}\left(\xi_{1}\right) \otimes \psi_{n+1}\right|_{V^{*}}\left(\xi_{2}\right) \otimes \cdots \otimes \psi_{n+l-1}\right|_{V^{*}}\left(\xi_{l}\right)
$$

for $\xi_{i} \in V^{*}$. By definition, $\psi_{n}(\xi \otimes \eta)=\psi_{n}(\xi) \psi_{n+l}(\eta)$ for all $l, m, n \in \mathbb{Z}$ and $\xi \in$ $\left(V^{*}\right)^{\otimes l}, \eta \in\left(V^{*}\right)^{\otimes m}$. If $\lambda=\sum_{i} \xi_{i 1} \otimes \xi_{i 2} \otimes \cdots \otimes \xi_{i h} \in R^{\perp}$, then for any $f=\sum_{i} v_{i 1} \otimes$ $v_{i 2} \otimes \cdots \otimes v_{i h} \in R^{\prime}$,

$$
\begin{aligned}
& \left(\left.\left.\left.\sum_{i} \psi_{n}\right|_{V^{*}}\left(\xi_{i 1}\right) \otimes \psi_{n+1}\right|_{V^{*}}\left(\xi_{i 2}\right) \otimes \cdots \otimes \psi_{n+h-1}\right|_{V^{*}}\left(\xi_{i h}\right)\right)(f) \\
& \quad=\sum_{i}\left(\left.\psi_{n}\right|_{V^{*}}\left(\xi_{i 1}\right)\right)\left(v_{i 1}\right) \otimes\left(\left.\psi_{n+1}\right|_{V^{*}}\left(\xi_{i 2}\right)\right)\left(v_{i 2}\right) \otimes \cdots \otimes\left(\left.\psi_{n+h-1}\right|_{V^{*}}\left(\xi_{i h}\right)\right)\left(v_{i h}\right) \\
& =\sum_{i} \xi_{i 1}\left(\left.\phi_{n}\right|_{V}\left(v_{i 1}\right)\right) \otimes \xi_{i 2}\left(\left.\phi_{n+1}\right|_{V}\left(v_{i 2}\right)\right) \otimes \cdots \otimes \xi_{i h}\left(\left.\phi_{n+h-1}\right|_{V}\left(v_{i h}\right)\right) \\
& =\lambda\left(\left.\left.\left.\sum_{i} \phi_{n}\right|_{V}\left(v_{i 1}\right) \otimes \phi_{n+1}\right|_{V}\left(v_{i 2}\right) \otimes \cdots \otimes \phi_{n+h-1}\right|_{V}\left(v_{i h}\right)\right)=0,
\end{aligned}
$$

so $\left.\left.\left.\sum_{i} \psi_{n}\right|_{V^{*}}\left(\xi_{i 1}\right) \otimes \psi_{n+1}\right|_{V^{*}}\left(\xi_{i 2}\right) \otimes \cdots \otimes \psi_{n+h-1}\right|_{V^{*}}\left(\xi_{i h}\right) \in R^{\prime \perp}$. Moreover, let $\xi \otimes \lambda \otimes$ $\eta \in\left(V^{*}\right)^{\otimes s} \otimes\left(V^{*}\right)^{\otimes h} \otimes\left(V^{*}\right)^{\otimes t}$ for $s, t \geq 0$. Then $\xi \otimes \lambda \otimes \eta \in\left(V^{*}\right)^{\otimes s} \otimes R^{\perp} \otimes\left(V^{*}\right)^{\otimes t}$ if and only if $\psi_{n}(\xi) \otimes \psi_{n+s}(\lambda) \otimes \psi_{n+s+h}(\eta) \in\left(V^{*}\right)^{\otimes s} \otimes R^{\perp} \otimes\left(V^{*}\right)^{\otimes t}$. Since

$$
\begin{aligned}
A_{i}^{!} & =\frac{\left(V^{*}\right)^{\otimes i}}{\sum_{s+t+h=i}\left(V^{*}\right)^{\otimes s} \otimes R^{\perp} \otimes\left(V^{*}\right)^{\otimes t}}, \\
\left(A^{\prime}\right)_{i}^{!} & =\frac{\left(V^{*}\right)^{\otimes i}}{\sum_{s+t+h=i}\left(V^{*}\right)^{\otimes s} \otimes R^{\perp} \otimes\left(V^{*}\right)^{\otimes t}},
\end{aligned}
$$

as $k$-vector spaces for all $i \geq h$, graded vector space isomorphisms $\psi_{n}: A^{!} \rightarrow\left(A^{\prime}\right)^{!}$ are well-defined. By construction, $\psi_{n}(a b)=\psi_{n}(a) \psi_{n+l}(b)$ for all $l, m, n \in \mathbb{Z}$ and $a \in$ $A_{l}^{!}, b \in A_{m}^{!}$, so $A^{!}$is isomorphic to a twist of $\left(A^{\prime}\right)^{!}$by [20, Proposition 2.8]. Hence $\operatorname{GrMod} A^{!} \cong \operatorname{GrMod}\left(A^{\prime}\right) !$ by [20, Theorem 1.2].

We now define Koszul algebras and graded Frobenius algebras.

Definition 4.2. Let $A$ be a connected graded algebra, and suppose $k \in \operatorname{GrMod} A$ has a minimal free resolution of the form

$$
\cdots \rightarrow \bigoplus_{j=1}^{r_{i}} A\left(-s_{i j}\right) \rightarrow \cdots \rightarrow \bigoplus_{j=1}^{r_{0}} A\left(-s_{0 j}\right) \rightarrow k \rightarrow 0 .
$$

The complexity of $A$ is defined by

$$
c_{A}:=\inf \left\{d \in \mathbb{R}^{+} \mid r_{i} \leq c i^{d-1} \text { for some constant } c>0, i \gg 0\right\} .
$$

We say that $A$ is Koszul if $s_{i j}=i$ for all $1 \leq j \leq r_{i}$ and all $i \in \mathbb{N}$. 
It is known that if $A$ is Koszul, then $A$ is quadratic, and its dual graded algebra $A^{!}$ is also Koszul, which is called the Koszul dual of $A$. In this case, $c_{A}=\operatorname{GKdim} A^{!}$.

Definition 4.3. A graded algebra $A$ is called a graded Frobenius algebra of Gorenstein parameter $\ell$ if $A^{*} \cong A_{v^{-1}}(-\ell)$ as graded $A$ - $A$ bimodules for some graded algebra automorphism $v \in \underline{\text { Aut }}_{k} A$, called the Nakayama automorphism of $A$. We say that $A$ is graded symmetric if $A^{*} \cong A(-\ell)$ as graded $A$ - $A$ bimodules.

Note that if $A$ is a connected graded Frobenius algebra, then $A$ is graded symmetric if and only if the Nakayama automorphism $v$ of $A$ is the identity. Let $A$ be a connected graded algebra. By [15, Proposition 5.10], $A$ is an AS-regular Koszul algebra of GKdimension $d$ if and only if $A^{!}$is a Frobenius Koszul algebra of complexity $d$, so classifying AS-regular Koszul algebras of GKdimension $d$ up to isomorphism of graded algebras (respectively, up to graded Morita equivalence) is equivalent to classifying Frobenius Koszul algebras of complexity $d$ up to isomorphism of graded algebras (respectively, up to graded Morita equivalence) by Lemma 4.1. In this case, if the Gorenstein parameter of $A$ is $\ell$, then it is easy to see that the global dimension of $A$ is $\ell$ and the Gorenstein parameter of $A^{!}$is $-\ell$.

Definition 4.4. A quadratic algebra $A$ is called co-geometric if $A^{!}=A(E, \sigma)$ is geometric. In this case, we write $A=A^{!}(E, \sigma)$.

THEOREM 4.5. Let $A=T(V) /(R)=A^{!}(E, \sigma)$ and $A^{\prime}=T(V) /\left(R^{\prime}\right)=A^{!}\left(E^{\prime}, \sigma^{\prime}\right)$ be co-geometric Frobenius Koszul algebras of finite complexities and of Gorenstein parameters $-d,-d^{\prime}$ with the Nakayama automorphisms $v \in \underline{\operatorname{Aut}}_{k} A, v^{\prime} \in \mathrm{Aut}_{k} A^{\prime}$. If $\operatorname{GrMod} A \cong \operatorname{GrMod} A^{\prime}$, then $d=d^{\prime}$ and $A^{!}\left(E, v \sigma^{d}\right) \cong A^{!}\left(E^{\prime}, v^{\prime}\left(\sigma^{\prime}\right)^{d}\right)$ as graded algebras.

Proof. If GrMod $A \cong \operatorname{GrMod} A^{\prime}$, then $\operatorname{GrMod} A^{!} \cong \operatorname{GrMod}\left(A^{\prime}\right)^{!}$by Lemma 4.1. Since $A^{!}=A(E, \sigma),\left(A^{\prime}\right)^{!}=A\left(E^{\prime}, \sigma^{\prime}\right)$ are geometric AS-regular Koszul algebras of global dimensions $d, d^{\prime}$, it follows that $d=d^{\prime}$, and the Gorenstein parameters of $A^{!},\left(A^{\prime}\right)^{!}$are both $d$. Moreover, since the generalised Nakayama automorphisms of $A^{\text {! }}$ and $\left(A^{\prime}\right)^{!}$are $(-1)^{d-1} v^{*} \in \underline{\operatorname{Aut}}_{k} A^{!}$and $(-1)^{d-1}\left(v^{\prime}\right)^{*} \in \underline{\operatorname{Aut}}_{k}\left(A^{\prime}\right)^{!}$by [17, Theorem 9.2], and they induce automorphisms $v \in \operatorname{Aut}_{k} E$ and $v^{\prime} \in \overline{A u t}_{k} E^{\prime}$,

$$
\begin{array}{ll} 
& \text { GrMod } A \cong \operatorname{GrMod} A^{\prime} \\
\Longleftrightarrow & \text { GrMod } A^{!} \cong \operatorname{GrMod}\left(A^{\prime}\right)^{!} \\
\Longrightarrow & A\left(E, v \sigma^{d}\right) \cong \overline{A^{!}} \cong \overline{\left(A^{\prime}\right)^{!} \cong A\left(E^{\prime}, v^{\prime}\left(\sigma^{\prime}\right)^{d}\right)} \\
\Longleftrightarrow & A^{!}\left(E, v \sigma^{d}\right) \cong \overline{A^{!}} \cong \overline{\left(A^{\prime}\right)^{!}} \cong A^{!}\left(E^{\prime}, v^{\prime}\left(\sigma^{\prime}\right)^{d}\right),
\end{array}
$$

by Lemma 4.1 and Theorem 3.5.

Corollary 4.6. Let $A=A^{!}(E, \sigma)$ and $A^{\prime}=A^{!}\left(E^{\prime}, \sigma^{\prime}\right)$ be Frobenius Koszul algebras of finite complexities and of Gorenstein parameter -3 with the Nakayama automorphisms $v \in \operatorname{Aut}_{k} A, v^{\prime} \in \operatorname{Aut}_{k} A^{\prime}$ such that $E \cong E^{\prime}$. Suppose that $E=\mathbb{P}^{2}$ or $E$ is a reduced and reducible cubic in $\mathbb{P}^{2}$. Then $\operatorname{GrMod} A \cong \operatorname{GrMod} A^{\prime}$ if and only if $A^{!}\left(E, v \sigma^{3}\right) \cong$ $A^{!}\left(E^{\prime}, v^{\prime}\left(\sigma^{\prime}\right)^{3}\right)$ as graded algebras.

A skew exterior algebra

$$
A=k\left\langle x_{1}, \ldots, x_{n}\right\rangle /\left(\alpha_{i j} x_{i} x_{j}+x_{j} x_{i}, x_{i}^{2}\right),
$$


where $\alpha_{i j} \in k$ such that $\alpha_{i j} \alpha_{j i}=\alpha_{i i}=1$ for $1 \leq i, j \leq n$ is a typical example of a Frobenius Koszul algebra. We apply our results to skew exterior algebras.

LEMMA 4.7. Let

$$
\begin{aligned}
& A=k\left\langle x_{1}, \ldots, x_{n}\right\rangle /\left(\alpha_{i j} x_{i} x_{j}+x_{j} x_{i}, x_{i}^{2}\right), \\
& A^{\prime}=k\left\langle x_{1}, \ldots, x_{n}\right\rangle /\left(\alpha_{i j}^{\prime} x_{i} x_{j}+x_{j} x_{i}, x_{i}^{2}\right)
\end{aligned}
$$

be skew exterior algebras.

(1) $A \cong A^{\prime}$ as graded algebras if and only if there exists a permutation $\theta \in S_{n}$ such that $\alpha_{i j}^{\prime}=\alpha_{\theta(i) \theta(j)}$ for $1 \leq i, j \leq n$.

(2) $\operatorname{GrMod} A \cong \operatorname{GrMod} A^{\prime}$ if and only if there exists a permutation $\theta \in S_{n}$ and $0 \neq m_{1}, \ldots, m_{n} \in k$ such that $\alpha_{i j}^{\prime}=m_{j} m_{i}^{-1} \alpha_{\theta(i) \theta(j)}$ for $1 \leq i, j \leq n$.

Proof. (1) This follows from Lemma 4.1 (1) and [19, Lemma 2.3].

(2) This follows from Lemma 4.1 (2) and [19, Theorem 2.5].

It is known that every (ungraded) Frobenius algebra that is Morita equivalent to symmetric algebra is symmetric. The situation in the graded case is different as the following theorem shows.

THEOREM 4.8. Every skew exterior algebra is graded Morita equivalent to a graded symmetric skew exterior algebra.

Proof. Let

$$
A=k\left\langle x_{1}, \ldots, x_{n}\right\rangle /\left(\alpha_{i j} x_{i} x_{j}+x_{j} x_{i}, x_{i}^{2}\right)
$$

be a skew exterior algebra and $\beta_{i}:=\alpha_{1 i} \alpha_{2 i} \cdots \alpha_{n i}$ for $1 \leq i \leq n$. Since $\beta_{1} \beta_{2} \cdots \beta_{n}=1$, we may choose $m_{i}=\sqrt[n]{\beta_{i}} \in k$ such that $m_{1} m_{2} \cdots m_{n}=1$. We define a new skew exterior algebra $A^{\prime}$ by

$$
A^{\prime}:=k\left\langle x_{1}, \ldots, x_{n}\right\rangle /\left(\alpha_{i j}^{\prime} x_{i} x_{j}+x_{j} x_{i}, x_{i}^{2}\right),
$$

where $\alpha_{i j}^{\prime}=m_{i} m_{j}^{-1} \alpha_{i j}$. By Lemma 4.7, GrMod $A \cong \operatorname{GrMod} A^{\prime}$. The Nakayama automorphism $v^{\prime}$ of $A^{\prime}$ is given by

$$
\begin{aligned}
v^{\prime}\left(x_{i}\right) & =(-1)^{n-1} \prod_{1 \leq t \leq n} \alpha_{i t}^{\prime} x_{i} \\
& =(-1)^{n-1} m_{i}^{n} m_{1}^{-1} m_{2}^{-1} \cdots m_{n}^{-1} \alpha_{i 1} \alpha_{i 2} \cdots \alpha_{i n} x_{i} \\
& =(-1)^{n-1} x_{i},
\end{aligned}
$$

for $1 \leq i \leq n$ (see the proof of [16, Theorem 4.1]), so if $n$ is odd, then $A^{\prime}$ is graded symmetric.

If $n$ is even, then define $m_{1}^{\prime}:=-m_{1}, m_{i}^{\prime}:=m_{i}$ for $2 \leq i \leq n$ and a new skew exterior algebra $A^{\prime \prime}$ by

$$
A^{\prime \prime}:=k\left\langle x_{1}, \ldots, x_{n}\right\rangle /\left(\alpha_{i j}^{\prime \prime} x_{i} x_{j}+x_{j} x_{i}, x_{i}^{2}\right)
$$


where $\alpha_{i j}^{\prime \prime}=m_{i}^{\prime}\left(m_{j}^{\prime}\right)^{-1} \alpha_{i j}$. By Lemma 4.7, GrMod $A \cong \operatorname{GrMod} A^{\prime \prime}$. The Nakayama automorphism $v^{\prime \prime}$ of $A^{\prime \prime}$ is given by

$$
\begin{aligned}
v^{\prime \prime}\left(x_{1}\right) & =(-1)^{n-1} \prod_{1 \leq t \leq n} \alpha_{1 t}^{\prime \prime} x_{1}=(-1)^{n-1}(-1)^{n+1} \prod_{1 \leq t \leq n} \alpha_{1 t}^{\prime} x_{1}=x_{1}, \\
v^{\prime \prime}\left(x_{i}\right) & =(-1)^{n-1} \prod_{1 \leq t \leq n} \alpha_{i t}^{\prime \prime} x_{i}=-(-1)^{n-1} \prod_{1 \leq t \leq n} \alpha_{i t}^{\prime} x_{i} \\
& =(-1)^{n} x_{i}=x_{i} \quad(2 \leq i \leq n),
\end{aligned}
$$

so $A^{\prime \prime}$ is graded symmetric.

4.2. Beilinson algebras. Finally, we apply our results to Beilinson algebras.

Definition 4.9. [7, 12]. Let $A$ be an AS-regular algebra of Gorenstein parameter $\ell$ or a graded Frobenius algebra of Gorenstein parameter $\ell$. The Beilinson algebra of $A$ is defined by

$$
\nabla A:=\left(\begin{array}{cccc}
A_{0} & A_{1} & \cdots & A_{r-1} \\
0 & A_{0} & \cdots & A_{r-2} \\
\vdots & \vdots & \ddots & \vdots \\
0 & 0 & \cdots & A_{0}
\end{array}\right)
$$

where $r=|\ell|$.

THEOREM 4.10. If $A$ and $A^{\prime}$ are AS-regular Koszul algebras, then the following are equivalent:

(1) $\operatorname{GrMod} A \cong \operatorname{GrMod} A^{\prime}$.

(2) $\nabla A \cong \nabla A^{\prime}$ as algebras.

(3) $\operatorname{GrMod} A^{!} \cong \operatorname{GrMod}\left(A^{\prime}\right)$ !

(4) $\nabla\left(A^{!}\right) \cong \nabla\left(\left(A^{\prime}\right)^{!}\right)$as algebras.

Proof. This follows from [7, Theorem 4.17, Theorem 4.24] and Lemma 4.1.

If $A$ is a $d$-dimensional AS-regular algebra, then $\nabla A$ is a $(d-1)$-dimensional quasi-Fano algebra introduced in [6], which is a nice class of a finite-dimensional algebra of global dimension $d-1$ (see [7] and [12] for details). By the above theorem, classifying $d$-dimensional AS-regular algebras up to graded Morita equivalence is the same as classifying Beilinson algebras of global dimension $d-1$ up to isomorphism. This observation is one of the motivations of this paper.

It is known that every finite-dimensional algebra of global dimension 1 is a path algebra of a finite acyclic quiver up to Morita equivalence, so such algebras can be classified in terms of quivers. As an obvious next step, it is interesting to classify finitedimensional algebras of global dimension 2. Such an algebra is a path algebra of a quiver with relations, and, in general, it is not easy to check if two algebras given as path algebras of quivers with relations are isomorphic as algebras by constructing an explicit algebra isomorphism. On the other hand, it is much easier to check if two graded algebras $T(V) / I$ and $T\left(V^{\prime}\right) / I^{\prime}$ generated in degree 1 over $k$ are isomorphic as graded algebras since any such isomorphism is induced by the vector space isomorphism $V \rightarrow V^{\prime}$. In this sense, the geometric techniques developed in this paper are useful 
for the classification of a class of finite-dimensional algebras of global dimension 2 , namely, Beilinson algebras of global dimension 2 .

EXAMPLE 4.11. Fix the Beilinson quiver

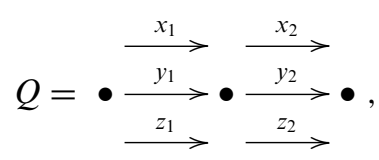

and let $B=k Q / I, B^{\prime}=k Q / I^{\prime}$ and $B^{\prime \prime}=k Q / I^{\prime \prime}$ be the path algebras with admissible relations (which must be generated by linear combinations of paths of length 2 )

$$
\begin{array}{ll}
I=\left(y_{1} z_{2}-\alpha z_{1} y_{2}, z_{1} x_{2}-\beta x_{1} z_{2}, x_{1} y_{2}-\gamma y_{1} x_{2}\right), & \alpha \beta \gamma \neq 0,1 \\
I^{\prime}=\left(y_{1} z_{2}-\alpha^{\prime} x_{1} x_{2}, z_{1} x_{2}-\beta^{\prime} y_{1} y_{2}, x_{1} y_{2}-\gamma^{\prime} z_{1} z_{2}\right), & \alpha^{\prime} \beta^{\prime} \gamma^{\prime} \neq 0,1 \\
I^{\prime \prime}=\left(y_{1} z_{2}-\alpha^{\prime \prime} z_{1} y_{2}-x_{1} x_{2}, z_{1} x_{2}-\beta^{\prime \prime} x_{1} z_{2}, x_{1} y_{2}-\beta^{\prime \prime} y_{1} x_{2}\right), \alpha^{\prime \prime}\left(\beta^{\prime \prime}\right)^{2} \neq 0,1 .
\end{array}
$$

It is easy to see that they are the Beilinson algebras of the following three-dimensional geometric AS-regular algebras

$$
\begin{aligned}
& A=A(E, \sigma)=k\langle x, y, z\rangle /(y z-\alpha z y, z x-\beta x z, x y-\gamma y x), \\
& A^{\prime}=A\left(E^{\prime}, \sigma^{\prime}\right)=k\langle x, y, z\rangle /\left(y z-\alpha^{\prime} x^{2}, z x-\beta^{\prime} y^{2}, x y-\gamma^{\prime} z^{2}\right), \\
& A^{\prime \prime}=A\left(E^{\prime \prime}, \sigma^{\prime \prime}\right)=k\langle x, y, z\rangle /\left(y z-\alpha^{\prime \prime} z y-x^{2}, z x-\beta^{\prime \prime} x z, x y-\beta^{\prime \prime} y x\right)
\end{aligned}
$$

of types $S_{1}, S_{3}$ and $S_{1}^{\prime}$, respectively. It is not easy to see directly whether or not there exists an algebra isomorphism among $B, B^{\prime}$ and $B^{\prime \prime}$. However, since $E \cong E^{\prime} ¥ E^{\prime \prime}$, we see that

$$
\operatorname{GrMod} A^{\prime \prime} ¥ \operatorname{GrMod} A \text { and } \operatorname{GrMod} A^{\prime \prime} ¥ \operatorname{GrMod} A^{\prime}
$$

by Lemma 2.5 (2), so we can conclude that

$$
B^{\prime \prime} ¥ B \text { and } B^{\prime \prime} ¥ B^{\prime}
$$

as algebras for any choice of parameters by Theorem 4.10. Moreover, it is not difficult to compute

$$
\begin{aligned}
& \bar{A}=k\langle x, y, z\rangle /(y z-\alpha \beta \gamma z y, z x-\alpha \beta \gamma x z, x y-\alpha \beta \gamma y x), \\
& \overline{A^{\prime}}=k\langle x, y, z\rangle /\left(\alpha^{\prime} \beta^{\prime} \gamma^{\prime} y z-z y, \alpha^{\prime} \beta^{\prime} \gamma^{\prime} z x-x z, \alpha^{\prime} \beta^{\prime} \gamma^{\prime} x y-y x\right) .
\end{aligned}
$$

Since $\bar{A}$ and $\overline{A^{\prime}}$ are skew polynomial algebras, it is easy to check when they are isomorphic as graded algebras [19, Lemma 2.3]. Using Theorem 4.10 and Theorem 3.9, the following are equivalent:

(1) $B \cong B^{\prime}$ as algebras.

(2) $\operatorname{GrMod} A \cong \operatorname{GrMod} A^{\prime}$.

(3) $\bar{A} \cong \overline{A^{\prime}}$ as graded algebras.

(4) $\alpha^{\prime} \beta^{\prime} \gamma^{\prime}=(\alpha \beta \gamma)^{ \pm 1}$.

ACKNOWLEDGEMENTS. The first author was supported by Grant-in-Aid for Scientific Research (C) 91540020. 


\section{REFERENCES}

1. M. Artin and W. Schelter, Graded algebras of global dimension 3, Adv. Math. 66 (1987), $171-216$.

2. M. Artin, J. Tate and M. Van den Bergh, Some algebras associated to automorphisms of elliptic curves in The Grothendieck Festschrift volume 1, Progress in Mathematics, vol. 86 (Birkhauser, Boston, 1990), 33-85.

3. M. Artin, J. Tate and M. Van den Bergh, Modules over regular algebras of dimension 3, Invent. Math. 106 (1991), 335-388. 228-287.

4. M. Artin and J. J. Zhang, Noncommutative projective schemes, Adv. Math. 109 (1994),

5. P. Jørgensen, Local cohomology for non-commutative graded algebras, Commun. Algebra 25 (1997), 575-591. $67-101$.

6. H. Minamoto, Ampleness of two-sided tilting complexes, Int. Math. Res. Not. 1 (2012),

7. H. Minamoto and I. Mori, The structure of AS-Gorenstein algebras, Adv. Math. 226 (2011), 4061-4095.

8. I. Mori, Noncommutative projective schemes and point schemes in Algebras, rings and their representations (World Scientific, Hackensack, NJ, 2006), 215-239. 656.

9. I. Mori, Co-point modules over Koszul algebras, J. London Math. Soc. 74 (2006), 639-

10. I. Mori, Co-point modules over Frobenius Koszul algebras, Commun. Algebra 36 (2008), $4659-4677$.

11. I. Mori, Classification problems in noncommutative algebraic geometry and representation theory in Representations of algebras and related topics, EMS Series of Congress Reports (Eur. Math. Soc., Zurich, 2011), 355-406.

12. I. Mori, B-cosntrcution and C-construction, Commun. Algebra, to appear.

13. K. de Naeghel and M. Van den Bergh, Ideal classes of three-dimensional Sklyanin algebras, J. Algebra 276 (2004), 515-551.

14. M. Nafari, M. Vancliff and J. Zhang, Classifying quadratic quantum $\mathbb{P}^{2} \mathrm{~s}$ by using graded skew Clifford algebras, J. Algebra 346 (2011), 152-164.

15. S. P. Smith, Some finite dimensional algebras related to elliptic curves in Representation theory of algebras and related topics (Mexico City, 1994), CMS Conference Proceedings, vol. 19 (Amer. Math. Soc., Providence, RI, 1996), 315-348.

16. K. Ueyama, Graded Morita equivalences for generic Artin-Schelter regular algebras, Kyoto J. Math. 51 (2011), 485-501.

17. M. Van den Bergh, Existence theorems for dualizing complexes over non-commutative graded and filtered rings, J. Algebra 195 (1997), 662-679.

18. K. Van Rompay, Segre product of Artin-Schelter regular algebras of dimension 2 and embeddings in quantum $\mathbb{P}^{3}$ 's, J. Algebra 180 (1996), 483-512.

19. J. Vitoria, Equivalences for noncommutative projective spaces, (2011), arXiv: $1001.4400 \mathrm{v} 3$.

20. J. J. Zhang, Twisted graded algebras and equivalences of graded categories, Proc. London Math. Soc. 72 (1996), 281-311. 